Article

\title{
Three-Step Projective Methods for Solving the Split Feasibility Problems
}

\author{
Suthep Suantai ${ }^{1}$, Nontawat Eiamniran ${ }^{2}$, Nattawut Pholasa ${ }^{3}$ and Prasit Cholamjiak ${ }^{3, *}$ \\ 1 Data Science Research Center, Department of Mathematics, Faculty of Science, Chiang Mai University, \\ Chiang Mai 50200, Thailand \\ 2 Demonstration School, University of Phayao, Phayao 56000, Thailand \\ 3 School of Science, University of Phayao, Phayao 56000, Thailand \\ * Correspondence: prasit.ch@up.ac.th
}

Received: 30 June 2019; Accepted: 31 July 2019; Published: 6 August 2019

check for updates

\begin{abstract}
In this paper, we focus on studying the split feasibility problem (SFP) in Hilbert spaces. Based on the CQ algorithm involving the self-adaptive technique, we introduce a three-step iteration process for approximating the solution of SFP. Then, the convergence results are established under mild conditions. Numerical experiments are provided to show the efficiency in signal processing. Some comparisons to various methods are also provided in this paper.
\end{abstract}

Keywords: self-adaptive technique; split feasibility problem; convergence theorems; Hilbert space; CQ algorithm

\section{Introduction}

In the present work, we aim to study the split feasibility problem (SFP), which is to find a point

$$
x^{*} \in C \text { such that } A x^{*} \in Q \text {, }
$$

where $C$ and $Q$ are non-empty, closed, and convex subsets of $\mathbb{R}^{M}$ and $\mathbb{R}^{N}$, and $A$ is an $M \times N$ matrix. The SFP was first investigated in 1994 by Censor-Elfving [1]. Subsequently, Xu [2,3] also studied this problem in finite dimensional Hilbert spaces. There have also been real-world applications, such as image processing and signal recovery.

Censor et al. [4] (see also [5]) introduced the Split Inverse Problem (SIP). In this, let $X$ and $Y$ be two vector spaces and $A: X \rightarrow Y$ be a linear operator, such that two inverse problems are involved. Denote IP1 and IP2 by such inverse problems in $X$ and $Y$, respectively. Given these data, the SIP is formulated as follows: find a point $x^{*} \in X$ that solves IP1, and such that the point $y^{*}=A x^{*} \in Y$ solves IP2.

It is known that the special case of the SFP can be reformulated to the following constrained minimization:

$$
\min _{x \in C}\left\|P_{Q}(A x)-A x\right\|
$$

Due to this reformulation, it can be seen as the following linear equation:

$$
x^{*} \in C \text { and } A x^{*}=b .
$$

In 2002, Byrne [6,7] introduced a new projection algorithm for the SFP. It was defined as follows:

$$
x_{n+1}=P_{C}\left(x_{n}-\tau_{n} A^{*}\left(I-P_{Q}\right) A x_{n}\right)
$$


where $P_{C}$ and $P_{Q}$ are projections onto $C$ and $Q$, and $A^{*}$ denotes the adjoint operator of $A$. This method is often called the CQ algorithm. In this case, the convergence is guaranteed when the step-size $\tau_{n}$ is in $\left(0, \frac{2}{\|A\|^{2}}\right)$, where $\|A\|^{2}$ is the spectral radius of the operator $A^{*} A$ and $I$ stands for the identity operator. However, it should be noted that projections are not easy to be calculated, and also come with costs of computation.

In practical applications, the sets $C$ and $Q$ are usually defined by

$$
C=\left\{x \in H_{1}: c(x) \leq 0\right\} \text { and } Q=\left\{y \in H_{2}: q(y) \leq 0\right\},
$$

where $c: H_{1} \rightarrow \mathbb{R}$ and $q: H_{2} \rightarrow \mathbb{R}$ are convex and sub-differential functions on $H_{1}$ and $H_{2}$. We always assume that $\partial c$ and $\partial q$ are bounded operators.

In 2004, Yang [8] presented the relaxed CQ algorithm, which follows from the idea of Fukushima [9]. The relaxed CQ algorithm, $P_{C}$ and $P_{Q}$, has been replaced by $P_{C_{n}}$ and $P_{Q_{n}}$, respectively, where $C_{n}$ and $Q_{n}$ are defined by

$$
C_{n}=\left\{x \in H_{1}: c\left(x_{n}\right) \leq\left\langle\xi_{n}, x_{n}-x\right\rangle\right\},
$$

where $\xi_{n} \in \partial c\left(x_{n}\right)$ and

$$
Q_{n}=\left\{y \in H_{2}: q\left(A x_{n}\right) \leq\left\langle\zeta_{n}, A x_{n}-y\right\rangle\right\},
$$

where $\zeta_{n} \in \partial q\left(A x_{n}\right)$. It is easily seen that $C_{n} \supset C$ and $Q_{n} \supset Q$ for all $n \geq 1$. Next, we set

$$
f_{n}(x)=\frac{1}{2}\left\|\left(I-P_{Q_{n}}\right) A x\right\|^{2}, n \geq 1 .
$$

In this case, we get

$$
\nabla f_{n}(x)=A^{*}\left(I-P_{Q_{n}}\right) A x .
$$

Since these sets are half-spaces, the computation for these projections is easy. However, if the step-size depends on the norm of operators, it is not an easy task to undertake. In fact, the relaxed CQ algorithm in a finite-dimentional Hilbert space was introduced by Yang [8] as follows:

$$
x_{n+1}=P_{C_{n}}\left(x_{n}-\tau_{n} \nabla f_{n}\left(x_{n}\right)\right),
$$

where $\tau_{n} \in\left(0,2 /\|A\|^{2}\right)$. We note that the norm of $A$ turned out to be costly in the computation. In particular, $A$ is a dense matrix and has a large dimension.

To overcome this difficultly, in 2012, López et al. [10] presented a new step-size $\tau_{n}$ as follows:

$$
\tau_{n}=\frac{\rho_{n} f_{n}\left(x_{n}\right)}{\left\|\nabla f_{n}\left(x_{n}\right)\right\|^{2}}
$$

where $\left\{\rho_{n}\right\}$ is a sequence in $(0,4)$ such that $\inf _{n \in \mathbb{N}} \rho_{n}\left(4-\rho_{n}\right)>0$. It was shown that $\left\{x_{n}\right\}$, with the step-size (11), converged weakly to a solution of SFP.

Another algorithm that can produce strong convergence is the Halpern-type algorithm. It is defined by

$$
x_{n+1}=\alpha_{n} u+\left(1-\alpha_{n}\right) P_{C_{n}}\left(x_{n}-\tau_{n} \nabla f_{n}\left(x_{n}\right)\right),
$$

where $u \in H_{1}$ is fixed and $\tau_{n}$ is defined by (11). It was claimed that $\left\{x_{n}\right\}$ converges strongly to $P_{S} u$ when $\alpha_{n} \rightarrow 0$ and $\sum_{n=1}^{\infty} \alpha_{n}=\infty$.

Recently, in 2005, Qu-Xiu [11] suggested the relaxed CQ algorithm by using Armijo-linesearch in Euclidean spaces, and then Gibali et al. [12] generalized the results of Qu-Xiu [11] to real Hilbert spaces as follows: 


$$
\begin{aligned}
x_{n+1} & =P_{C_{n}}\left(x_{n}-\tau_{n} \nabla f_{n}\left(y_{n}\right)\right) \\
y_{n} & =P_{C_{n}}\left(x_{n}-\tau_{n} \nabla f_{n}\left(x_{n}\right)\right),
\end{aligned}
$$

where $\sigma>0, \rho, \mu \in(0,1), \tau_{n}=\sigma \rho^{\mu_{n}}$, and $\mu_{n}$ is the smallest non-negative integer, such that

$$
\tau_{n}\left\|\nabla f_{n}\left(x_{n}\right)-\nabla f_{n}\left(y_{n}\right)\right\| \leq \mu\left\|x_{n}-y_{n}\right\| .
$$

It was shown that $\left\{x_{n}\right\}$ converges weakly to a solution of SFP. Various iterative methods have been established to solve the SFP and some related problems—see, for example, [2-5,13-17].

We aim to suggest a new three-step iteration process by using the CQ algorithm with step-sizes that employ the self-adaptive terminology. We remark that our assumptions do not depend on the operator norms, which is an easy task in practice. We then establish weak and strong convergence results under suitable conditions. Finally, we apply our results to compressed sensing. Some comparisons are also given to those of Yang [8], Gibali et al. [12], and López et al. [10].

Moreover, based on the three-step iterative methods, some convergence results, including its efficiency, have been established-see, for example, [18-23].

\section{Basic Concepts}

We next recall some useful basic concepts that will be used in our proof. Let $H$ be a real Hilbert space. Let $T: H \rightarrow H$ be a nonlinear mapping. Then, $T$ is called

(i) nonexpansive if

$$
\|T x-T y\| \leq\|x-y\|, \text { for all } x, y \in H .
$$

(ii) firmly nonexpansive if, for all $x, y \in H$,

$$
\|T x-T y\|^{2} \leq\langle x-y, T x-T y\rangle
$$

A function $f: H \rightarrow \mathbb{R}$ is convex if

$$
f(\lambda x+(1-\lambda) y) \leq \lambda f(x)+(1-\lambda) f(y), \text { for all } \lambda \in(0,1), \text { for all } x, y \in H
$$

A function $f: H \rightarrow \mathbb{R}$ is weakly lower semi-continuous (w-lsc) at $x$ if $x_{n} \rightarrow x$ implies

$$
f(x) \leq \liminf _{n \rightarrow \infty} f\left(x_{n}\right)
$$

The projection of a non-empty, closed, and convex set $C$ onto $H$ is defined by

$$
P_{C} x:=\arg \min _{y \in C}\|x-y\|^{2}, x \in H .
$$

We note that $P_{C}$ and $I-P_{C}$ are firmly non-expansive. From [7], we know that if

$$
f(x)=\frac{1}{2}\left\|\left(I-P_{Q}\right) A x\right\|^{2}
$$

then $\nabla f$ is $\|A\|^{2}$-Lipschitz continuous. Moreover, in real Hilbert spaces, we know that [24]

(i) $\quad\left\langle x-P_{C} x, z-P_{C} x\right\rangle \leq 0$ for all $z \in C$;

(ii) $\quad\left\|P_{C} x-P_{C} y\right\|^{2} \leq\left\langle P_{C} x-P_{C} y, x-y\right\rangle$ for all $x, y \in H$;

(iii) $\quad\left\|P_{C} x-z\right\|^{2} \leq\|x-z\|^{2}-\left\|P_{C} x-x\right\|^{2}$ for all $z \in C$.

Lemma 1. [25] Let $H$ be a real Hilbert space and $S$ be a non-empty, closed, and convex subset of $H$. Let $\left\{x_{n}\right\}$ be a sequence in $H$ that satisfies the following conditions: 
(i) For each $x \in S, \lim _{n \rightarrow \infty}\left\|x_{n}-x\right\|$ exists;

(ii) $\quad \omega_{w}\left(x_{n}\right) \subset S$.

Then, $\left\{x_{n}\right\}$ converges weakly to a point in $S$.

Lemma 2. [26] Let $\left\{s_{n}\right\}$ be a non-negative real sequence, such that

$$
\begin{aligned}
& s_{n+1} \leq\left(1-\alpha_{n}\right) s_{n}+\alpha_{n} \mu_{n}, n \geq 1, \\
& s_{n+1} \leq s_{n}-\lambda_{n}+v_{n}, n \geq 1,
\end{aligned}
$$

where $\left\{\alpha_{n}\right\} \subseteq(0,1),\left\{\lambda_{n}\right\}$ is a non-negative, real sequence, and $\left\{\mu_{n}\right\}$ and $\left\{v_{n}\right\}$ are real sequences such that

(i) $\quad \sum_{n=1}^{\infty} \alpha_{n}=\infty$;

(ii) $\quad \lim _{n \rightarrow \infty} v_{n}=0$;

(iii) $\lim _{k \rightarrow \infty} \lambda_{n_{k}}=0$ implies limsup $\mu_{n_{k}} \leq 0$ for any subsequence $\left\{n_{k}\right\}$ of $\{n\}$.

Then, $\lim _{n \rightarrow \infty} s_{n}=0$.

Next, we propose Algorithms 1 and 2 for solving the split feasibility problem in Hilbert spaces.

\section{Weak Convergence Result}

We next introduce a new CQ algorithm and derive the weak convergence of the proposed method.

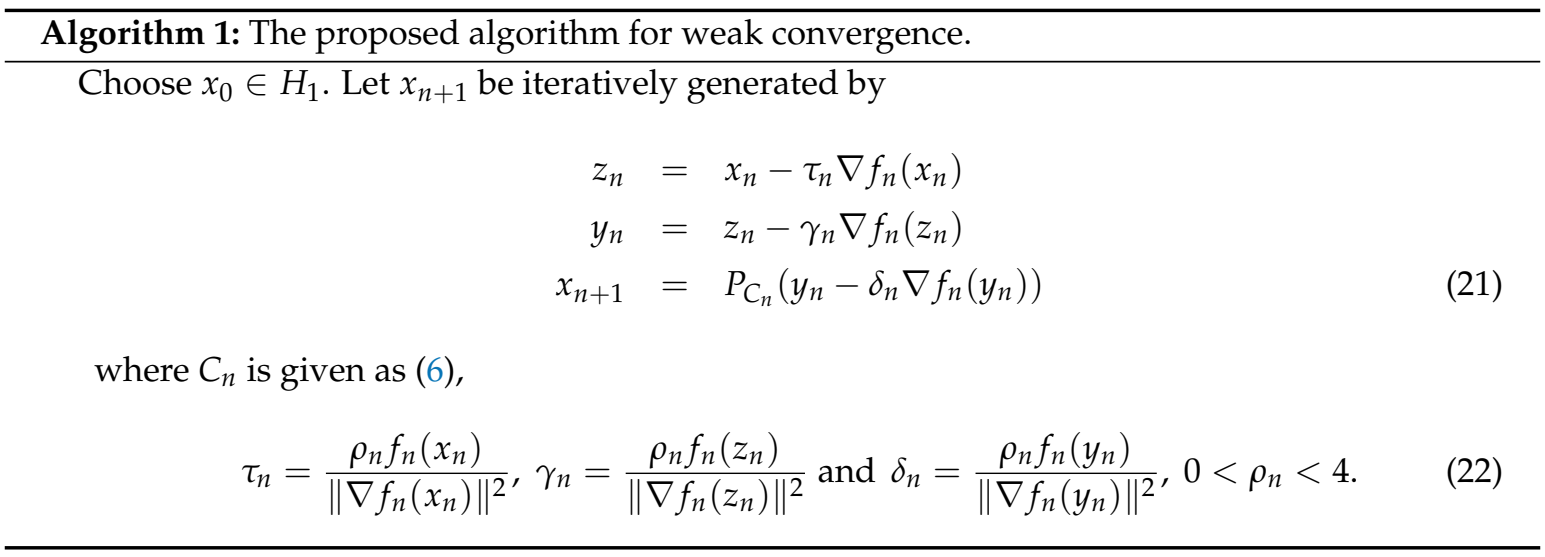

Remark 1. We see that Algorithm 1 is defined as the iterates $z_{n}$ and $y_{n}$ by a gradient method with the step-size $\tau_{n}$ and $\gamma_{n}$, respectively, and the iterate $x_{n+1}$ is defined by a relaxed CQ algorithm with the step-size $\delta_{n}$.

In this paper, we denote $S$ by the solution set of SFP and assume that $S$ is non-empty. Next, we prove its weak convergence theorem as follows:

Theorem 1. Suppose $\inf _{n} \rho_{n}\left(4-\rho_{n}\right)>0$. Then, $\left\{x_{n}\right\}$, defined by Algorithm 1, converges weakly to a point of S.

Proof. Let $\hat{x} \in S$. Because $C \subseteq C_{n}$ and $Q \subseteq Q_{n}$, we have $\hat{x}=P_{C}(\hat{x})=P_{C_{n}}(\hat{x})$ and $A \hat{x}=P_{Q}(A \hat{x})=P_{Q_{n}}(A \hat{x})$. It follows that $\nabla f_{n}(\hat{x})=0$. Then we obtain 


$$
\begin{aligned}
\left\|x_{n+1}-\hat{x}\right\|^{2}= & \left\|P_{C_{n}}\left(y_{n}-\delta_{n} \nabla f_{n}\left(y_{n}\right)\right)-\hat{x}\right\|^{2} \\
\leq & \left\|y_{n}-\delta_{n} \nabla f_{n}\left(y_{n}\right)-\hat{x}\right\|^{2}-\left\|x_{n+1}-y_{n}+\delta_{n} \nabla f_{n}\left(y_{n}\right)\right\|^{2} \\
= & \left\|y_{n}-\hat{x}\right\|^{2}+\delta_{n}^{2}\left\|\nabla f_{n}\left(y_{n}\right)\right\|^{2}-2 \delta_{n}\left\langle y_{n}-\hat{x}, \nabla f_{n}\left(y_{n}\right)\right\rangle \\
& -\left\|x_{n+1}-y_{n}+\delta_{n} \nabla f_{n}\left(y_{n}\right)\right\|^{2} .
\end{aligned}
$$

From (23) and $\nabla f_{n}(\hat{x})=0$, we see that

$$
\begin{aligned}
\left\langle y_{n}-\hat{x}, \nabla f_{n}\left(y_{n}\right)\right\rangle & =\left\langle y_{n}-\hat{x}, \nabla f_{n}\left(y_{n}\right)-\nabla f_{n}(\hat{x})\right\rangle \\
& =\left\langle y_{n}-\hat{x}, A^{*}\left(I-P_{Q_{n}}\right) A y_{n}-A^{*}\left(I-P_{Q_{n}}\right) A \hat{x}\right\rangle \\
& =\left\langle A y_{n}-A \hat{x},\left(I-P_{Q_{n}}\right) A y_{n}-\left(I-P_{Q_{n}}\right) A \hat{x}\right\rangle \\
& \geq\left\|\left(I-P_{Q_{n}}\right) A y_{n}\right\|^{2} \\
& =2 f_{n}\left(y_{n}\right) .
\end{aligned}
$$

We can also show that

$$
\left\langle x_{n}-\hat{x}, \nabla f_{n}\left(x_{n}\right)\right\rangle \geq 2 f_{n}\left(x_{n}\right)
$$

and

$$
\left\langle z_{n}-\hat{x}, \nabla f_{n}\left(z_{n}\right)\right\rangle \geq 2 f_{n}\left(z_{n}\right) .
$$

So, by (26), it follows that

$$
\begin{aligned}
\left\|y_{n}-\hat{x}\right\|^{2} & =\left\|z_{n}-\gamma_{n} \nabla f_{n}\left(z_{n}\right)-\hat{x}\right\|^{2} \\
& =\left\|z_{n}-\hat{x}\right\|^{2}+\gamma_{n}^{2}\left\|\nabla f_{n}\left(z_{n}\right)\right\|^{2}-2 \gamma_{n}\left\langle z_{n}-\hat{x}, \nabla f_{n}\left(z_{n}\right)\right\rangle \\
& \leq\left\|z_{n}-\hat{x}\right\|^{2}+\gamma_{n}^{2}\left\|\nabla f_{n}\left(z_{n}\right)\right\|^{2}-4 \gamma_{n} f_{n}\left(z_{n}\right) .
\end{aligned}
$$

Moreover, by (25), we obtain

$$
\begin{aligned}
\left\|z_{n}-\hat{x}\right\|^{2} & =\left\|x_{n}-\tau_{n} \nabla f_{n}\left(x_{n}\right)-\hat{x}\right\|^{2} \\
& =\left\|x_{n}-\hat{x}\right\|^{2}+\tau_{n}^{2}\left\|\nabla f_{n}\left(x_{n}\right)\right\|^{2}-2 \tau_{n}\left\langle x_{n}-\hat{x}, \nabla f_{n}\left(x_{n}\right)\right\rangle \\
& \leq\left\|x_{n}-\hat{x}\right\|^{2}+\tau_{n}^{2}\left\|\nabla f_{n}\left(x_{n}\right)\right\|^{2}-4 \tau_{n} f_{n}\left(x_{n}\right) .
\end{aligned}
$$

Combining (23)-(28), we have

$$
\begin{aligned}
\left\|x_{n+1}-\hat{x}\right\|^{2} \leq & \left\|x_{n}-\hat{x}\right\|^{2}+\tau_{n}^{2}\left\|\nabla f_{n}\left(x_{n}\right)\right\|^{2}-4 \tau_{n} f_{n}\left(x_{n}\right)+\gamma_{n}^{2}\left\|\nabla f_{n}\left(z_{n}\right)\right\|^{2} \\
& -4 \gamma_{n} f_{n}\left(z_{n}\right)+\delta_{n}^{2}\left\|\nabla f_{n}\left(y_{n}\right)\right\|^{2}-4 \delta_{n} f_{n}\left(y_{n}\right)-\left\|x_{n+1}-y_{n}+\delta_{n} \nabla f_{n}\left(y_{n}\right)\right\|^{2} \\
= & \left\|x_{n}-\hat{x}\right\|^{2}+\frac{\rho_{n}^{2} f_{n}^{2}\left(x_{n}\right)}{\left(\left\|\nabla f_{n}\left(x_{n}\right)\right\|^{2}\right)^{2}}\left\|\nabla f_{n}\left(x_{n}\right)\right\|^{2}-\frac{4 \rho_{n} f_{n}^{2}\left(x_{n}\right)}{\left\|\nabla f_{n}\left(x_{n}\right)\right\|^{2}} \\
& +\frac{\rho_{n}^{2} f_{n}^{2}\left(z_{n}\right)}{\left(\left\|\nabla f_{n}\left(z_{n}\right)\right\|^{2}\right)^{2}}\left\|\nabla f_{n}\left(z_{n}\right)\right\|^{2}-\frac{4 \rho_{n} f_{n}^{2}\left(z_{n}\right)}{\left\|\nabla f_{n}\left(z_{n}\right)\right\|^{2}}+\frac{\rho_{n}^{2} f_{n}^{2}\left(y_{n}\right)}{\left(\left\|\nabla f_{n}\left(y_{n}\right)\right\|^{2}\right)^{2}}\left\|\nabla f_{n}\left(y_{n}\right)\right\|^{2} \\
& \quad-\frac{4 \rho_{n} f_{n}^{2}\left(y_{n}\right)}{\left\|\nabla f_{n}\left(y_{n}\right)\right\|^{2}}-\left\|x_{n+1}-y_{n}+\delta_{n} \nabla f_{n}\left(y_{n}\right)\right\|^{2} \\
= & \left\|x_{n}-\hat{x}\right\|^{2}+\frac{\rho_{n}^{2} f_{n}^{2}\left(x_{n}\right)}{\left\|\nabla f_{n}\left(x_{n}\right)\right\|^{2}}-\frac{4 \rho_{n} f_{n}^{2}\left(x_{n}\right)}{\left\|\nabla f_{n}\left(x_{n}\right)\right\|^{2}}+\frac{\rho_{n}^{2} f_{n}^{2}\left(z_{n}\right)}{\left\|\nabla f_{n}\left(z_{n}\right)\right\|^{2}}-\frac{4 \rho_{n} f_{n}^{2}\left(z_{n}\right)}{\left\|\nabla f_{n}\left(z_{n}\right)\right\|^{2}} \\
& +\frac{\rho_{n}^{2} f_{n}^{2}\left(y_{n}\right)}{\left\|\nabla f_{n}\left(y_{n}\right)\right\|^{2}}-\frac{4 \rho_{n} f_{n}^{2}\left(y_{n}\right)}{\left\|\nabla f_{n}\left(y_{n}\right)\right\|^{2}}-\left\|x_{n+1}-y_{n}+\delta_{n} \nabla f_{n}\left(y_{n}\right)\right\|^{2} \\
= & \left\|x_{n}-\hat{x}\right\|^{2}-\rho_{n}\left(4-\rho_{n}\right) \frac{f_{n}^{2}\left(x_{n}\right)}{\left\|\nabla f_{n}\left(x_{n}\right)\right\|^{2}}-\rho_{n}\left(4-\rho_{n}\right) \frac{f_{n}^{2}\left(z_{n}\right)}{\left\|\nabla f_{n}\left(z_{n}\right)\right\|^{2}} \\
& -\rho_{n}\left(4-\rho_{n}\right) \frac{f_{n}^{2}\left(y_{n}\right)}{\left\|\nabla f_{n}\left(y_{n}\right)\right\|^{2}}-\left\|x_{n+1}-y_{n}+\delta_{n} \nabla f_{n}\left(y_{n}\right)\right\|^{2} .
\end{aligned}
$$


This implies that, since $0<\rho_{n}<4$,

$$
\left\|x_{n+1}-\hat{x}\right\| \leq\left\|x_{n}-\hat{x}\right\| .
$$

Thus, $\lim _{n \rightarrow \infty}\left\|x_{n}-\hat{x}\right\|$ exists and $\left\{x_{n}\right\}$ is bounded. Since $\inf _{n \in \mathbb{N}} \rho_{n}\left(4-\rho_{n}\right)>0$, there is a $\rho$ such that $\rho_{n}\left(4-\rho_{n}\right) \geq \rho(4-\rho)>0$. Again, from (29), it yields

$$
\begin{aligned}
\left\|x_{n}-\hat{x}\right\|^{2}-\left\|x_{n+1}-\hat{x}\right\|^{2} \geq & \rho(4-\rho) \frac{f_{n}^{2}\left(x_{n}\right)}{\left\|\nabla f_{n}\left(x_{n}\right)\right\|^{2}}+\rho(4-\rho) \frac{f_{n}^{2}\left(z_{n}\right)}{\left\|\nabla f_{n}\left(z_{n}\right)\right\|^{2}}+\rho(4-\rho) \frac{f_{n}^{2}\left(y_{n}\right)}{\left\|\nabla f_{n}\left(y_{n}\right)\right\|^{2}} \\
& +\left\|x_{n+1}-y_{n}+\delta_{n} \nabla f_{n}\left(y_{n}\right)\right\|^{2} .
\end{aligned}
$$

So, we obtain

$$
\begin{aligned}
0=\lim _{n \rightarrow \infty}\left\|x_{n+1}-\hat{x}\right\|^{2}-\left\|x_{n}-\hat{x}\right\|^{2} \geq & \lim _{n \rightarrow \infty}\left[\rho(4-\rho) \frac{f_{n}^{2}\left(x_{n}\right)}{\left\|\nabla f_{n}\left(x_{n}\right)\right\|^{2}}+\rho(4-\rho) \frac{f_{n}^{2}\left(z_{n}\right)}{\left\|\nabla f_{n}\left(z_{n}\right)\right\|^{2}}\right. \\
& \left.+\rho(4-\rho) \frac{f_{n}^{2}\left(y_{n}\right)}{\left\|\nabla f_{n}\left(y_{n}\right)\right\|^{2}}+\left\|x_{n+1}-y_{n}+\delta_{n} \nabla f_{n}\left(y_{n}\right)\right\|^{2}\right] \\
\geq & 0 .
\end{aligned}
$$

This shows that

$$
\begin{array}{r}
\lim _{n \rightarrow \infty} \frac{f_{n}^{2}\left(x_{n}\right)}{\left\|\nabla f_{n}\left(x_{n}\right)\right\|^{2}}=0, \\
\lim _{n \rightarrow \infty} \frac{f_{n}^{2}\left(z_{n}\right)}{\left\|\nabla f_{n}\left(z_{n}\right)\right\|^{2}}=0, \\
\lim _{n \rightarrow \infty} \frac{f_{n}^{2}\left(y_{n}\right)}{\left\|\nabla f_{n}\left(y_{n}\right)\right\|^{2}}=0, \\
\lim _{n \rightarrow \infty}\left\|x_{n+1}-y_{n}+\delta_{n} \nabla f_{n}\left(y_{n}\right)\right\|^{2}=0 .
\end{array}
$$

We can check that $\left\{\left\|\nabla f_{n}\left(x_{n}\right)\right\|\right\}$ is bounded. So $\lim _{n \rightarrow \infty} f_{n}\left(x_{n}\right)=0$. This means $\lim _{n \rightarrow \infty}\left\|\left(I-P_{Q_{n}}\right) A x_{n}\right\|=0$. Also $\lim _{n \rightarrow \infty} f_{n}\left(z_{n}\right)=\lim _{n \rightarrow \infty}\left\|\left(I-P_{Q_{n}}\right) A z_{n}\right\|=0$ and $\lim _{n \rightarrow \infty} f_{n}\left(y_{n}\right)=\lim _{n \rightarrow \infty} \|(I-$ $\left.P_{Q_{n}}\right) A y_{n} \|=0$.

Furthermore, from (33), we get

$$
\lim _{n \rightarrow \infty}\left\|x_{n+1}-y_{n}+\delta_{n} \nabla f_{n}\left(y_{n}\right)\right\|=0 .
$$

We note that

$$
\delta_{n}\left\|\nabla f_{n}\left(y_{n}\right)\right\|=\frac{\rho_{n} f_{n}\left(y_{n}\right)}{\left\|\nabla f_{n}\left(y_{n}\right)\right\|^{2}}\left\|\nabla f_{n}\left(y_{n}\right)\right\| \rightarrow 0, \text { as } n \rightarrow \infty .
$$

Hence, by (34) and (35), $\lim _{n \rightarrow \infty}\left\|x_{n+1}-y_{n}\right\|=0$. Further, by (21) and $\tau_{n}\left\|\nabla f_{n}\left(x_{n}\right)\right\| \rightarrow 0$ as $n \rightarrow \infty$, we get $\lim _{n \rightarrow \infty}\left\|z_{n}-x_{n}\right\|=0$. Since $\gamma_{n}\left\|\nabla f_{n}\left(x_{n}\right)\right\| \rightarrow 0$ as $n \rightarrow \infty$, we also get $\lim _{n \rightarrow \infty}\left\|y_{n}-z_{n}\right\|=0$. Hence $\lim _{n \rightarrow \infty}\left\|x_{n+1}-x_{n}\right\|=0$.

By the boundedness of $\left\{x_{n}\right\}$, the set $\omega_{w}\left(x_{n}\right)$ is non-empty. Let $x^{*} \in \omega_{w}\left(x_{n}\right)$. Then, there is a subsequence $\left\{x_{n_{k}}\right\}$ of $\left\{x_{n}\right\}$ that $x_{n_{k}} \rightarrow x^{*} \in H_{1}$.

Next, we show that $x^{*}$ is in $S$. Since $x_{n_{k}+1} \in C_{n_{k}}$, by the definition of $C_{n_{k}}$, we get

$$
c\left(x_{n_{k}}\right) \leq\left\langle\xi_{n_{k}}, x_{n_{k}}-x_{n_{k}+1}\right\rangle
$$

where $\xi_{n_{k}} \in \partial c\left(x_{n_{k}}\right)$. It follows, by the boundedness of $\partial c$, that

$$
\begin{aligned}
c\left(x_{n_{k}}\right) & \leq\left\|\xi_{n_{k}}\right\|\left\|x_{n_{k}}-x_{n_{k}+1}\right\| \\
& \rightarrow 0, \text { as } k \rightarrow \infty .
\end{aligned}
$$


By the w-lsc of $c, x_{n_{k}} \rightarrow x^{*}$ and (37), we see that

$$
c\left(x^{*}\right) \leq \liminf _{k \rightarrow \infty} c\left(x_{n_{k}}\right) \leq 0 .
$$

Thus, $x^{*} \in C$.

Next, we will show that $A x^{*} \in Q$. Since $P_{Q_{n_{k}}}\left(A x_{n_{k}}\right) \in Q_{n_{k}}$,

$$
q\left(A x_{n_{k}}\right) \leq\left\langle\eta_{n_{k}}, A x_{n_{k}}-P_{Q_{n_{k}}}\left(A x_{n_{k}}\right)\right\rangle
$$

where $\eta_{n_{k}} \in \partial q\left(A x_{n_{k}}\right)$. So, we obtain

$$
\begin{aligned}
q\left(A x_{n_{k}}\right) & \leq\left\|\eta_{n_{k}}\right\|\left\|A x_{n_{k}}-P_{Q_{n_{k}}}\left(A x_{n_{k}}\right)\right\| \\
& \rightarrow 0, \text { as } k \rightarrow \infty .
\end{aligned}
$$

The w-lsc of $q$ and (40) give that

$$
q\left(A x^{*}\right) \leq \liminf _{k \rightarrow \infty} q\left(A x_{n_{k}}\right) \leq 0 .
$$

Thus, $A x^{*} \in Q$. By Lemma 1 , we can deduce that $\left\{x_{n}\right\}$ converges weakly to a point in $S$.

\section{Strong Convergence Result}

We next discuss the strong convergence of the sequence generated by the Halpern-type iteration.

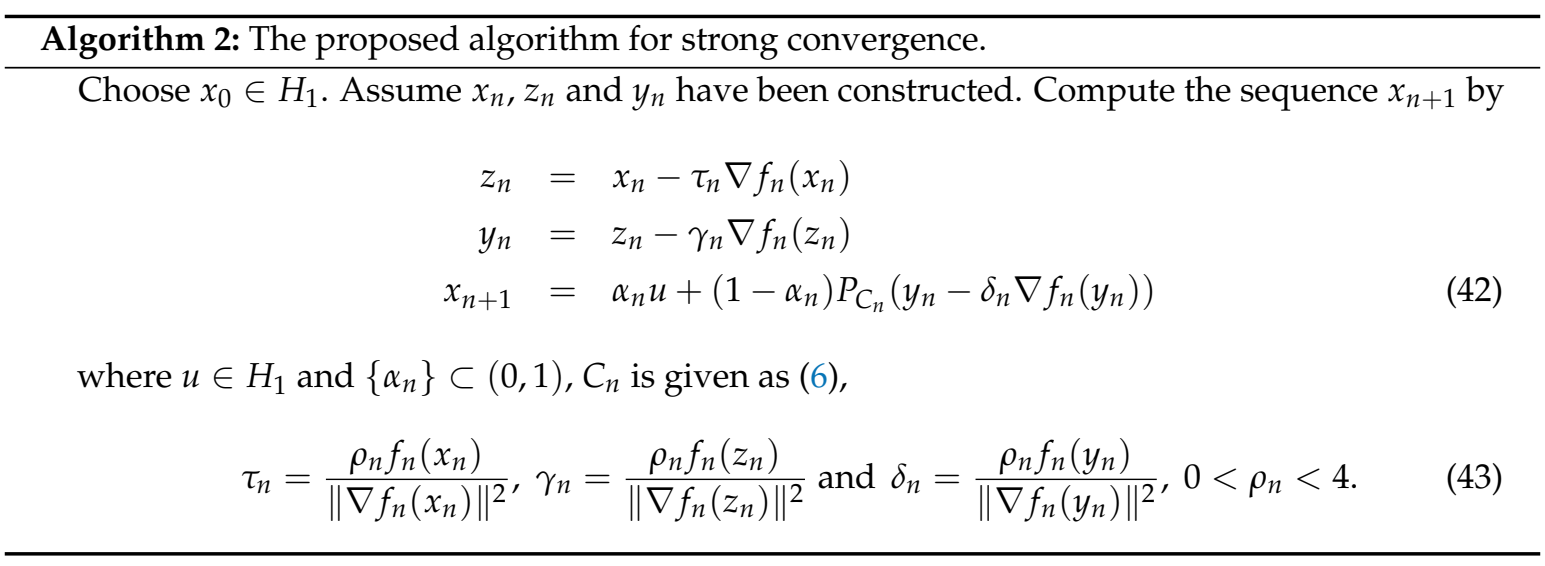

Theorem 2. Assume that $\left\{\alpha_{n}\right\}$ and $\left\{\rho_{n}\right\}$ satisfy the conditions:

(a) $\lim _{n \rightarrow \infty} \alpha_{n}=0$ and $\sum_{n=1}^{\infty} \alpha_{n}=\infty$;

(b) $\quad \inf _{n} \rho_{n}\left(4-\rho_{n}\right)>0$.

Then, $\left\{x_{n}\right\}$, defined by Algorithm 2, converges strongly to $P_{S} u$.

Proof. Set $\hat{x}=P_{S} u$. By using the same argument as in Theorem 1, we can show that

$$
\begin{aligned}
\left\|P_{C_{n}}\left(y_{n}-\delta_{n} \nabla f_{n}\left(y_{n}\right)\right)-\hat{x}\right\|^{2} \leq & \left\|y_{n}-\hat{x}\right\|^{2}-\rho_{n}\left(4-\rho_{n}\right) \frac{f_{n}^{2}\left(y_{n}\right)}{\left\|\nabla f_{n}\left(y_{n}\right)\right\|} \\
& -\left\|P_{C_{n}}\left(y_{n}-\delta_{n} \nabla f_{n}\left(y_{n}\right)\right)-y_{n}+\delta_{n} \nabla f_{n}\left(y_{n}\right)\right\|^{2} .
\end{aligned}
$$


So,

$$
\left\|y_{n}-\hat{x}\right\|^{2} \leq\left\|z_{n}-\hat{x}\right\|^{2}-\rho_{n}\left(4-\rho_{n}\right) \frac{f_{n}^{2}\left(z_{n}\right)}{\left\|\nabla f_{n}\left(z_{n}\right)\right\|^{2}}
$$

and

$$
\left\|z_{n}-\hat{x}\right\|^{2} \leq\left\|x_{n}-\hat{x}\right\|^{2}-\rho_{n}\left(4-\rho_{n}\right) \frac{f_{n}^{2}\left(x_{n}\right)}{\left\|\nabla f_{n}\left(x_{n}\right)\right\|^{2}}
$$

Also, we obtain

$$
\begin{aligned}
\left\|x_{n+1}-\hat{x}\right\|^{2} & =\left\|\alpha_{n}(u-\hat{x})+\left(1-\alpha_{n}\right)\left(P_{C_{n}}\left(y_{n}-\delta_{n} \nabla f_{n}\left(y_{n}\right)\right)-\hat{x}\right)\right\|^{2} \\
& \leq\left(1-\alpha_{n}\right)\left\|P_{C_{n}}\left(y_{n}-\delta_{n} \nabla f_{n}\left(y_{n}\right)\right)-\hat{x}\right\|^{2}+2 \alpha_{n}\left\langle u-\hat{x}, x_{n+1}-\hat{x}\right\rangle .
\end{aligned}
$$

Combining (44)-(47), we obtain

$$
\begin{aligned}
\left\|x_{n+1}-\hat{x}\right\|^{2} \leq & \left(1-\alpha_{n}\right)\left\|x_{n}-\hat{x}\right\|^{2}-\left(1-\alpha_{n}\right) \rho_{n}\left(4-\rho_{n}\right) \frac{f_{n}^{2}\left(x_{n}\right)}{\left\|\nabla f_{n}\left(x_{n}\right)\right\|^{2}} \\
& -\left(1-\alpha_{n}\right) \rho_{n}\left(4-\rho_{n}\right) \frac{f_{n}^{2}\left(z_{n}\right)}{\left\|\nabla f_{n}\left(z_{n}\right)\right\|^{2}}-\left(1-\alpha_{n}\right) \rho_{n}\left(4-\rho_{n}\right) \frac{f_{n}^{2}\left(y_{n}\right)}{\left\|\nabla f_{n}\left(y_{n}\right)\right\|^{2}} \\
& -\left(1-\alpha_{n}\right)\left\|P_{C_{n}}\left(y_{n}-\delta_{n} \nabla f_{n}\left(y_{n}\right)\right)-y_{n}+\delta_{n} \nabla f_{n}\left(y_{n}\right)\right\|^{2} \\
& +2 \alpha_{n}\left\langle u-\hat{x}, x_{n+1}-\hat{x}\right\rangle .
\end{aligned}
$$

Next, we will show that $\left\{x_{n}\right\}$ is bounded. Again, using (44)-(46), we get

$$
\begin{aligned}
\left\|x_{n+1}-\hat{x}\right\| & =\left\|\alpha_{n} u+\left(1-\alpha_{n}\right) P_{C_{n}}\left(y_{n}-\delta_{n} \nabla f_{n}\left(y_{n}\right)\right)-\hat{x}\right\| \\
& \leq \alpha_{n}\|u-\hat{x}\|+\left(1-\alpha_{n}\right)\left\|y_{n}-\hat{x}\right\| \\
& \leq \alpha_{n}\|u-\hat{x}\|+\left(1-\alpha_{n}\right)\left\|z_{n}-\hat{x}\right\| \\
& \leq \alpha_{n}\|u-\hat{x}\|+\left(1-\alpha_{n}\right)\left\|x_{n}-\hat{x}\right\| .
\end{aligned}
$$

This shows that $\left\{x_{n}\right\}$ is bounded. From Lemma 2 and (48), we set

$$
\begin{aligned}
s_{n}= & \left\|x_{n}-\hat{x}\right\|^{2} ; \\
v_{n}= & 2 \alpha_{n}\left\langle u-\hat{x}, x_{n+1}-\hat{x}\right\rangle ; \\
\mu_{n}= & 2\left\langle u-\hat{x}, x_{n+1}-\hat{x}\right\rangle ; \\
\lambda_{n}= & \left(1-\alpha_{n}\right)\left\|P_{C_{n}}\left(y_{n}-\delta_{n} \nabla f_{n}\left(y_{n}\right)\right)-y_{n}+\delta_{n} \nabla f_{n}\left(y_{n}\right)\right\|^{2}+\left(1-\alpha_{n}\right) \rho_{n}\left(4-\rho_{n}\right) \frac{f_{n}^{2}\left(x_{n}\right)}{\left\|\nabla f_{n}\left(x_{n}\right)\right\|^{2}} \\
& +\left(1-\alpha_{n}\right) \rho_{n}\left(4-\rho_{n}\right) \frac{f_{n}^{2}\left(y_{n}\right)}{\left\|\nabla f_{n}\left(y_{n}\right)\right\|^{2}}+\left(1-\alpha_{n}\right) \rho_{n}\left(4-\rho_{n}\right) \frac{f_{n}^{2}\left(z_{n}\right)}{\left\|\nabla f_{n}\left(z_{n}\right)\right\|^{2}} .
\end{aligned}
$$

So (48) can be transformed to the inequalities

$$
\begin{aligned}
& s_{n+1} \leq\left(1-\alpha_{n}\right) s_{n}+\alpha_{n} \mu_{n}, n \geq 1 \\
& s_{n+1} \leq s_{n}-\lambda_{n}+v_{n} .
\end{aligned}
$$

Let $\left\{n_{k}\right\}$ be a subsequence of $\{n\}$, such that

$$
\lim _{k \rightarrow \infty} \lambda_{n_{k}}=0
$$


Then, we have

$$
\begin{aligned}
& \lim _{k \rightarrow \infty}\left(1-\alpha_{n_{k}}\right)\left\|P_{C_{n_{k}}}\left(y_{n_{k}}-\delta_{n_{k}} \nabla f_{n_{k}}\left(y_{n_{k}}\right)\right)-y_{n_{k}}+\delta_{n_{k}} \nabla f_{n_{k}}\left(y_{n_{k}}\right)\right\|^{2} \\
& \quad+\left(1-\alpha_{n_{k}}\right) \rho_{n_{k}}\left(4-\rho_{n_{k}}\right) \frac{f_{n_{k}}^{2}\left(x_{n_{k}}\right)}{\left\|\nabla f_{n_{k}}\left(x_{n_{k}}\right)\right\|^{2}}+\left(1-\alpha_{n_{k}}\right) \rho_{n_{k}}\left(4-\rho_{n_{k}}\right) \frac{f_{n_{k}}^{2}\left(z_{n_{k}}\right)}{\left\|\nabla f_{n_{k}}\left(z_{n_{k}}\right)\right\|^{2}} \\
& \quad+\left(1-\alpha_{n_{k}}\right) \rho_{n_{k}}\left(4-\rho_{n_{k}}\right) \frac{f_{n_{k}}^{2}\left(y_{n_{k}}\right)}{\left\|\nabla f_{n_{k}}\left(y_{n_{k}}\right)\right\|^{2}}=0
\end{aligned}
$$

which implies by our assumptions that

$$
\begin{gathered}
\frac{f_{n_{k}}^{2}\left(x_{n_{k}}\right)}{\left\|\nabla f_{n_{k}}\left(x_{n_{k}}\right)\right\|^{2}} \rightarrow 0, \frac{f_{n_{k}}^{2}\left(z_{n_{k}}\right)}{\left\|\nabla f_{n_{k}}\left(z_{n_{k}}\right)\right\|^{2}} \rightarrow 0, \frac{f_{n_{k}}^{2}\left(y_{n_{k}}\right)}{\left\|\nabla f_{n_{k}}\left(y_{n_{k}}\right)\right\|^{2}} \rightarrow 0 \text { and } \\
\left\|P_{C_{n_{k}}}\left(y_{n_{k}}-\delta_{n_{k}} \nabla f_{n_{k}}\left(y_{n_{k}}\right)\right)-y_{n_{k}}+\delta_{n_{k}} \nabla f_{n_{k}}\left(y_{n_{k}}\right)\right\| \rightarrow 0
\end{gathered}
$$

as $k \rightarrow \infty$. Since $\left\{\left\|\nabla f_{n_{k}}\left(x_{n_{k}}\right)\right\|\right\},\left\{\left\|\nabla f_{n_{k}}\left(z_{n_{k}}\right)\right\|\right\}$ and $\left\{\left\|\nabla f_{n_{k}}\left(y_{n_{k}}\right)\right\|\right\}$ are bounded, it follows that $f_{n_{k}}\left(x_{n_{k}}\right) \rightarrow 0, f_{n_{k}}\left(z_{n_{k}}\right) \rightarrow 0$ and $f_{n_{k}}\left(y_{n_{k}}\right) \rightarrow 0$ as $k \rightarrow \infty$. We also get $\lim _{k \rightarrow \infty}\left\|\left(I-P_{Q_{n_{k}}}\right) A x_{n_{k}}\right\|=0$, $\lim _{k \rightarrow \infty}\left\|\left(I-P_{Q_{n_{k}}}\right) A z_{n_{k}}\right\|=0$ and $\lim _{k \rightarrow \infty}\left\|\left(I-P_{Q_{n_{k}}}\right) A y_{n_{k}}\right\|=0$.

As in Theorem 1, we can show that $\omega_{w}\left(x_{n_{k}}\right) \subset S$. Hence, there is a subsequence $\left\{x_{n_{k_{i}}}\right\}$ of $\left\{x_{n_{k}}\right\}$, such that $x_{n_{k_{i}}} \rightarrow x^{*} \in S$. So, we obtain

$$
\begin{aligned}
\limsup _{k \rightarrow \infty}\left\langle u-\hat{x}, x_{n_{k}}-\hat{x}\right\rangle & =\lim _{i \rightarrow \infty}\left\langle u-\hat{x}, x_{n_{k_{i}}}-\hat{x}\right\rangle \\
& =\left\langle u-\hat{x}, x^{*}-\hat{x}\right\rangle \\
& \leq 0
\end{aligned}
$$

On the other hand, we see that

$$
\begin{aligned}
\left\|x_{n_{k}+1}-y_{n_{k}}\right\|= & \left\|\alpha_{n_{k}} u+\left(1-\alpha_{n_{k}}\right) P_{C_{n_{k}}}\left(y_{n_{k}}-\delta_{n_{k}} \nabla f_{n_{k}}\left(y_{n_{k}}\right)\right)-y_{n_{k}}\right\| \\
\leq & \alpha_{n_{k}}\left\|u-y_{n_{k}}\right\|+\left(1-\alpha_{n_{k}}\right)\left\|P_{C_{n_{k}}}\left(y_{n_{k}}-\delta_{n_{k}} \nabla f_{n_{k}}\left(y_{n_{k}}\right)\right)-y_{n_{k}}\right\| \\
\leq & \alpha_{n_{k}}\left\|u-y_{n_{k}}\right\|+\left(1-\alpha_{n_{k}}\right)\left\|P_{C_{n_{k}}}\left(y_{n_{k}}-\delta_{n_{k}} \nabla f_{n_{k}}\left(y_{n_{k}}\right)\right)-y_{n_{k}}+\delta_{n_{k}} \nabla f_{n_{k}}\left(y_{n_{k}}\right)\right\| \\
& +\left(1-\alpha_{n_{k}}\right) \delta_{n_{k}}\left\|\nabla f_{n_{k}}\left(y_{n_{k}}\right)\right\| \\
\rightarrow & 0 \text { as } k \rightarrow \infty .
\end{aligned}
$$

We see that

$$
\lim _{k \rightarrow \infty}\left\|z_{n_{k}}-x_{n_{k}}\right\|=0 \text { and } \lim _{k \rightarrow \infty}\left\|y_{n_{k}}-z_{n_{k}}\right\|=0
$$

Hence, we obtain

$$
\begin{aligned}
\left\|x_{n_{k}+1}-x_{n_{k}}\right\| & \leq\left\|x_{n_{k}+1}-y_{n_{k}}\right\|+\left\|y_{n_{k}}-z_{n_{k}}\right\|+\left\|z_{n_{k}}-x_{n_{k}}\right\| \\
& \rightarrow 0 \text { as } k \rightarrow \infty .
\end{aligned}
$$

By (54) and (56), we obtain

$$
\limsup _{k \rightarrow \infty}\left\langle u-\hat{x}, x_{n_{k}+1}-\hat{x}\right\rangle \leq 0
$$

Hence, we get

$$
\limsup _{k \rightarrow \infty} \mu_{n_{k}} \leq 0
$$

By Lemma 2, we can deduce that $\left\{x_{n}\right\}$ converges strongly to $\hat{x}=P_{S} u$. 


\section{Numerical Examples}

Finally, we provide numerical experiments of the compressed sensing in signal recovery. We demonstrate the performance of the relaxed CQ algorithms defined by Yang [8], López et al. [10], Gibali et al. [12] and our CQ algorithms. The compressed sensing can be modeled as the linear equation:

$$
y=A x+\varepsilon,
$$

where $x \in \mathbb{R}^{N}$ is a recovered vector with $m$ non-zero components, $y \in \mathbb{R}^{M}$ is the observed data with noisy $\varepsilon$, and $A: \mathbb{R}^{N} \rightarrow \mathbb{R}^{M}(M<N)$. It is noted that (59) can be seen as solving the LASSO problem:

$$
\min _{x \in \mathbb{R}^{N}} \frac{1}{2}\|y-A x\|^{2} \text { subject to }\|x\|_{1} \leq t,
$$

where $t>0$. In particular, in case $C=\left\{x \in \mathbb{R}^{N}:\|x\|_{1} \leq t\right\}$ and $Q=\{y\}$, the LASSO problem can be considered as the SFP (1). From this point of view, we can apply the CQ algorithm to solve (60).

In our experiment, one matrix $A \in \mathbb{R}^{M \times N}$ is generated from a normal distribution with mean zero and invariance one. The sparse vector $x \in \mathbb{R}^{N}$ is generated from uniform distribution in the interval $[-1,1]$ with $m$ nonzero elements. The observation $y$ is generated by white Gaussian noise with signal-to-noise ratio $\mathrm{SNR}=40$. Let $t=m$ and $x_{1}=0$.

The stopping criterion is defined by the mean square error (MSE):

$$
\operatorname{MSE}=\frac{1}{N}\|\hat{x}-x\|_{2}^{2}<10^{-5},
$$

where $\hat{x}$ is an approximated signal of $x$.

In what follows, let $\tau_{n}=\frac{1}{\|A\|^{2}}$ in the CQ algorithm (10) by Yang [8], $\tau_{n}=\frac{\rho_{n}\|A x-y\|^{2}}{2\left\|A^{T}(A x-y)\right\|^{2}}$ with $\rho_{n}=2$ in (11) of López et al. [10], $\tau_{n}$ defined by (14) with $\sigma=1, \rho=\mu=0.5$ in that of Gibali et al. [12] and $\tau_{n}, \gamma_{n}, \delta_{n}$ defined by (22) with $\rho_{n}=2$. The numerical results are reported as follows.

From Table 1 and Figures 1 and 2, we observe that the convergence behavior of Algorithm 1 outperforms those of Yang [8], López et al. [10], Gibali et al. [12]. Indeed, Algorithm 1 has less number of iterations than other methods.

Table 1. Number of iterations for Algorithm 1.

\begin{tabular}{ccccc}
\hline Case 1: $N=512, M=256$ & Yang (10) & López et al. (11) & Gibali et al. (13) & Algorithm 1 \\
\hline$m=10$ & 74 & 65 & 106 & 39 \\
$m=20$ & 217 & 184 & 246 & 111 \\
\hline Case 2 $: N=4096, M=2048$ & Yang (10) & López et al. (11) & Gibali et al. (13) & Algorithm 1 \\
\hline$m=100$ & 87 & 77 & 117 & 48 \\
$m=200$ & 184 & 156 & 220 & 94 \\
\hline
\end{tabular}




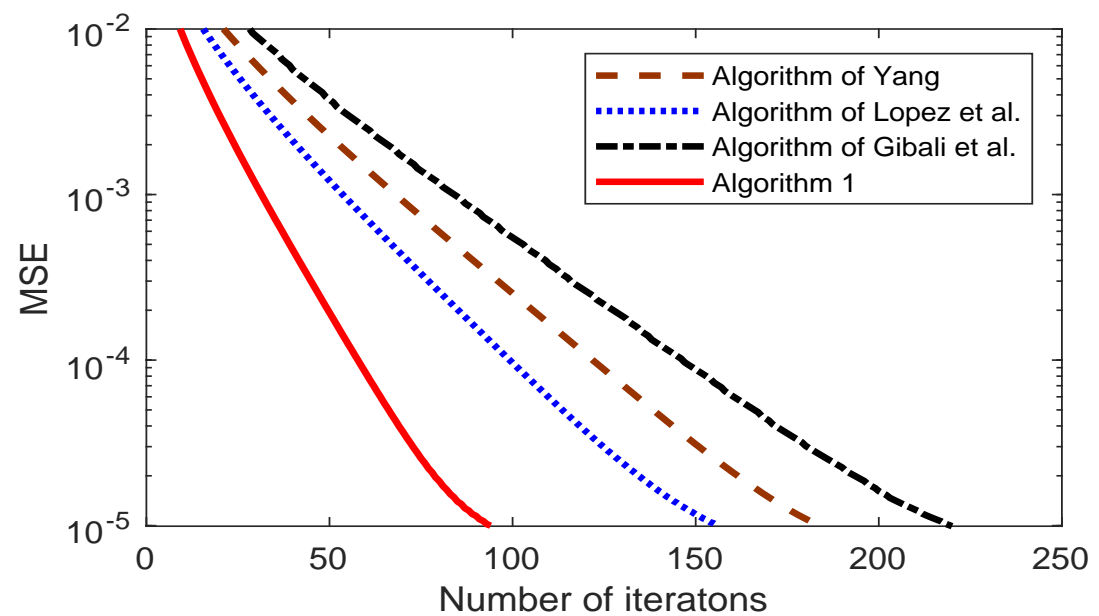

Figure 1. MSE versus number of iterations of Algorithm 1 in case $N=4096, M=2048$, and $m=200$.
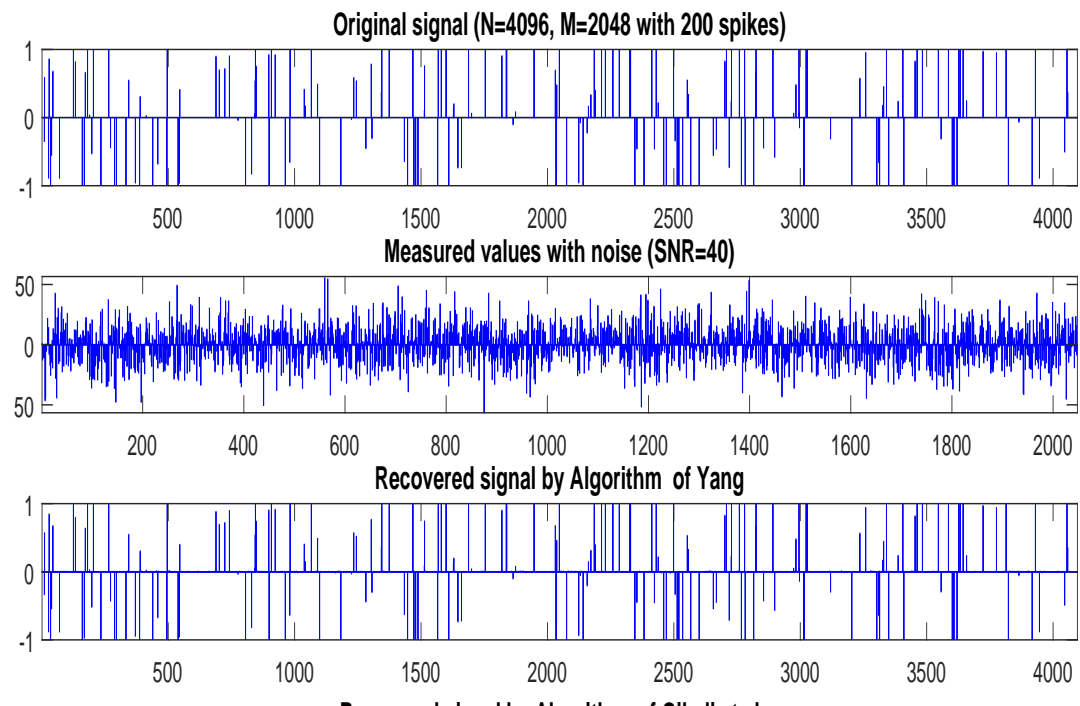

Recovered signal by Algorithm of Gibali et al.

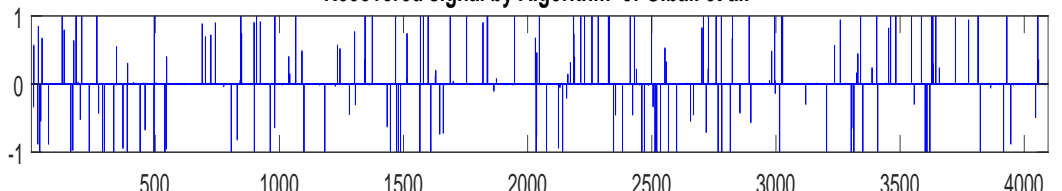

Recovered signal by Algorithm of Lopez et al.
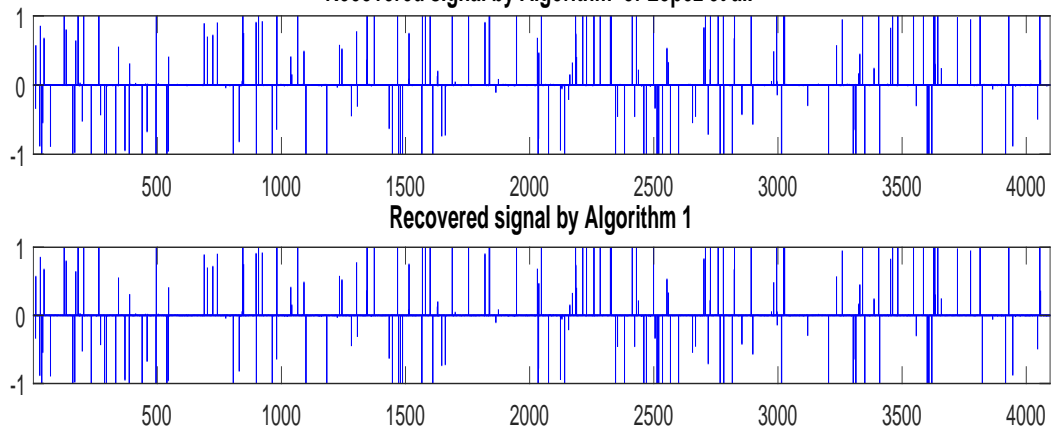

Figure 2. From top to bottom: original signal, observation data, recovered signal by Algorithms of Yang [8], López et al. [10], Gibali et al. [12], and Algorithm 1 with $N=4096, M=2048$ and $m=200$. 
Next, we discuss the strong convergence of the relaxed CQ algorithm (12) by López et al. [10] and Algorithm 2. We set each step-sizes $\tau_{n}$ as in the weak convergence and let $\alpha_{n}=\frac{1}{100 n+1}$. The initial vector $x_{1}=0$ and $u$ is generated randomly. Then, we have the following numerical results.

From Table 2 and Figures 3 and 4, it is observed that Algorithm 2 has a smaller number of iterations than that of López et al. [10].

Table 2. Number of iterations for Algorithm 2.

\begin{tabular}{ccc}
\hline Case $1: N=512, M=256$ & López et al. (12) & Algorithm 2 \\
\hline$m=10$ & 85 & 43 \\
$m=20$ & 119 & 64 \\
\hline Case 2 $N=4096, M=2048$ & López et al. (12) & Algorithm 2 \\
\hline$m=100$ & 85 & 48 \\
$m=200$ & 230 & 140 \\
\hline
\end{tabular}

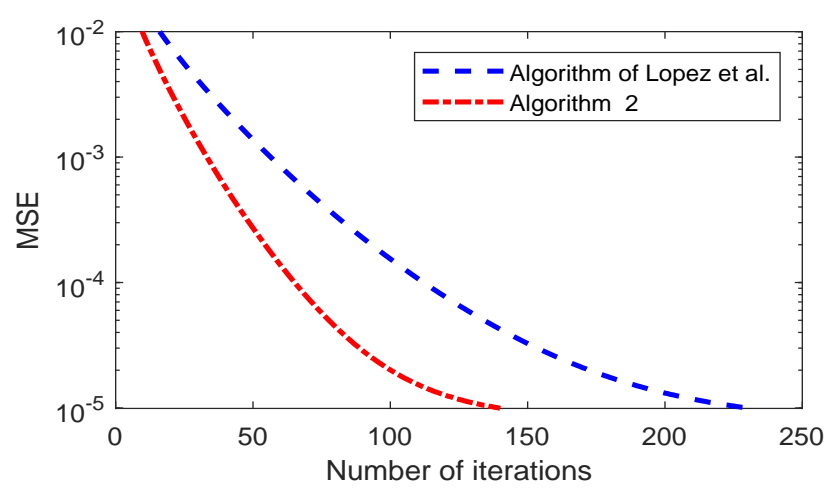

Figure 3. MSE versus number of iterations of Algorithm 2 in case $\mathrm{N}=4096, \mathrm{M}=2048$ and $\mathrm{m}=200$.
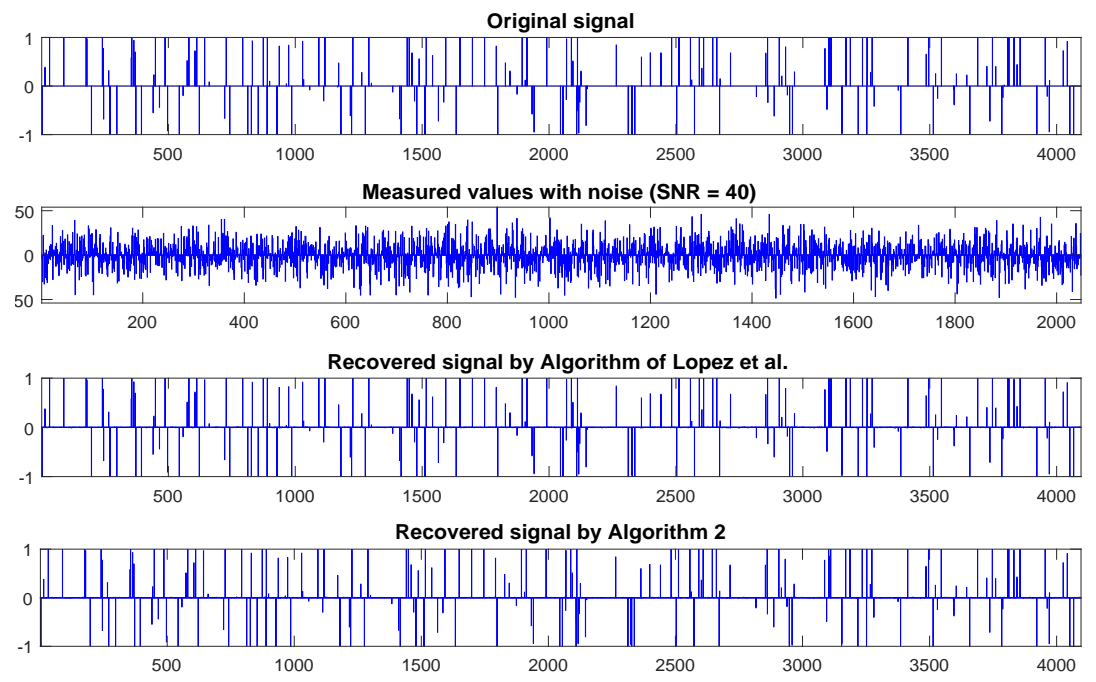

Figure 4. From top to bottom: original signal, observation data, recovered signal by Algorithms (12) of López et al. [10] and Algorithm 2.

We provide the numerical examples in $L_{2}$-space, which is an infinite Hilbert space, by using Algorithm 2. Let $H_{1}=H_{2}=L_{2}[0,1]$ with the inner product given by

$$
\langle f, g\rangle=\int_{0}^{1} f(t) g(t) d t .
$$


Let $C=\left\{x \in L_{2}[0,1]:\|x\|_{L_{2}} \leq 1\right\}$ and $Q=\left\{x \in L_{2}[0,1]:\left\langle x, \frac{t}{2}\right\rangle \leq 0\right\}$. Find $x \in C$ such that $A x \in Q$, where $(A x)(t)=\frac{x(t)}{2}$. We take $\alpha_{n}=\frac{1}{10 n+1}, \rho_{n}=1.75$. The stopping criterion is defined by

$$
E_{n}=\frac{1}{2}\left\|A x_{n}-P_{Q} A x_{n}\right\|_{L_{2}}^{2}<10^{-4} .
$$

From Table 3 and Figure 5, we see that our algorithm is better than that of López et al. [10] in terms of number of iterations and CPU time.

Table 3. Numberof iterations for Algorithm 2 in $L_{2}$-space.

\begin{tabular}{cccc}
\hline & & López et al. (12) & Algorithm 2 \\
\hline$u=t$ & No. of Iter. & 9 & 4 \\
$x_{1}=7 t^{2}+2$ & cpu (time) & 6.3707 & 4.0171 \\
\hline$u=t+1$ & No. of Iter. & 9 & 4 \\
$x_{1}=4 t^{2}+t+3$ & cpu (time) & 6.5169 & 4.1789 \\
\hline$u=t^{2}$ & No. of Iter. & 10 & 4 \\
$x_{1}=2 t^{2}+3 e^{t}$ & cpu (time) & 9.4818 & 5.5274 \\
\hline$u=t^{3}$ & No. of Iter. & 6 & 3 \\
$x_{1}=5 t^{3}+\sin (t)+1$ & cpu (time) & 3.7478 & 2.9404 \\
\hline
\end{tabular}
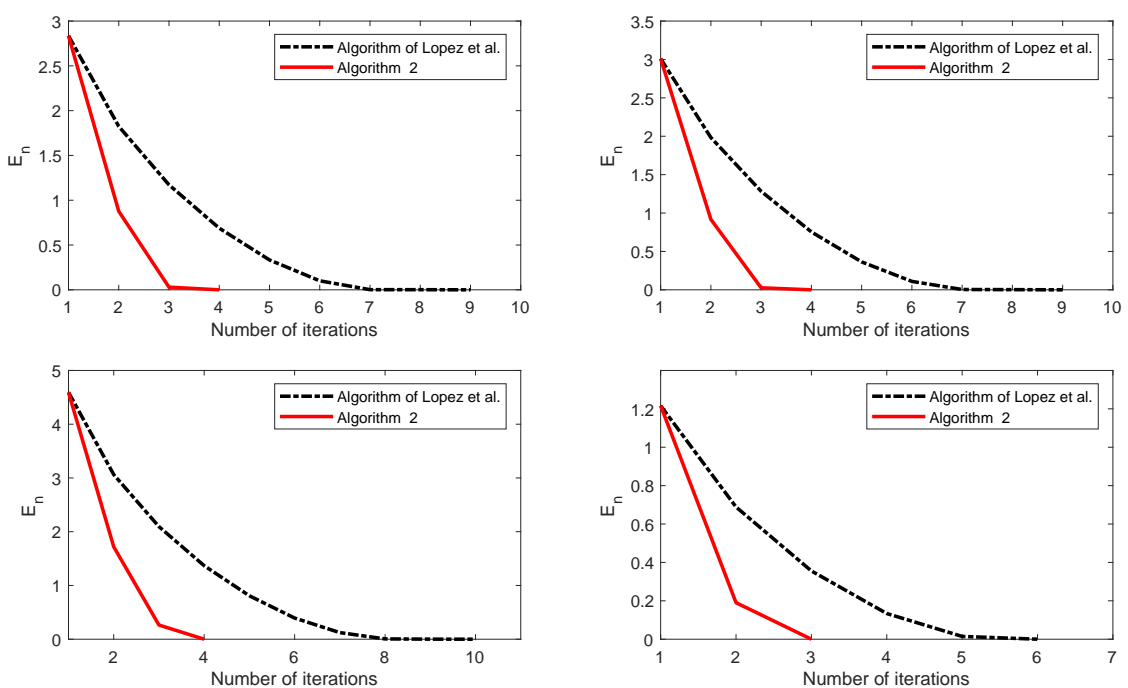

Figure 5. Error versus numberof iterations of Algorithm 2 in $L_{2}$-space.

\section{Conclusions}

In this work, we have introduced new three-step iterative methods involving the self-adaptive technique for the SFP in Hilbert spaces. Weak and strong convergence was discussed under suitable conditions. Preliminary numerical experiments showed that our proposed methods outperform those of Yang [8], López et al. [10], and Gibali et al. [12]. In future work, we aim to investigate the SFP in Banach spaces, and to also establish its convergence under suitable conditions. 
Author Contributions: S.S.; supervision and investigation, N.E.; writing original draft, N.P.; data analysis and P.C.; formal analysis and methodology.

Funding: This research is supported by Chiang Mai University.

Conflicts of Interest: The authors declare no conflict of interest.

\section{References}

1. Censor, Y.; Elfving, T. A multiprojection algorithms using Bregman projection in a product space. Numer. Algor. 1994, 8, 221-239. [CrossRef]

2. Wang, F.; Xu, H.K. Approximating curve and strong convergence of the CQ algorithm for the split feasibility problem. J. Inequal. Appl. 2010, 2010, 102085. [CrossRef]

3. $\mathrm{Xu}, \mathrm{H} . \mathrm{K}$. Iterative methods for the split feasibility problem in infinite-dimensional Hilbert spaces. Inverse Probl. 2010, 26, 105018. [CrossRef]

4. Censor, Y.; Gibali, A.; Reich, S. Algorithms for the split variational inequality problem. Numer. Algor. 2012, 59, 301-323. [CrossRef]

5. Gibali, A. A new split inverse problem and application to least intensity feasible solutions. Pure Appl. Funct. Anal. 2017, 2, 243-258.

6. Byrne, C. Iterative oblique projection onto convex sets and the split feasibility problem. Inverse Probl. 2002, 18, 441-453. [CrossRef]

7. Byrne, C. A unified treatment of some iterative algorithms in signal processing and image reconstruction. Inverse Probl. 2004, 20, 103-120. [CrossRef]

8. Yang, Q. The relaxed CQ algorithm for solving the split feasibility problem. Inverse Probl. 2004, 20, 1261-1266. [CrossRef]

9. Fukushima, M. A relaxed projection method for variational inequalities. Math. Program. 1986, 35, 58-70. [CrossRef]

10. López, G.; Martín-Márquez, V.; Wang, F.; Xu, H.K. Solving the split feasibility problem without prior knowledge of matrix norms. Inverse Probl. 2012, 28, 085004. [CrossRef]

11. Qu, B.; Xiu, N. A note on the CQ algorithm for the split feasibility problem. Inverse Probl. 2005, 21, 1655-1665. [CrossRef]

12. Gibali, A.; Liu, L.W.; Tang, Y.C. Note on the modified relaxation CQ algorithm for the split feasibility problem. Optim. Lett. 2017, 12, 1-14. [CrossRef]

13. Ćirić, L.; Rafiq, A.; Radenović, S.; Rajović, M.; Ume, J.S. On Mann implicit iterations for strongly accretive and strongly pseudo-contractive mappings. Appl. Math. Comput. 2008, 198, 128-137. [CrossRef]

14. Ćirić, L. Some Recent Results in Metrical Fixed Point Theory; University of Belgrade: Belgrade, Serbia, 2003.

15. Dang, Y.; Gao, Y. The strong convergence of a KM-CQ-like algorithm for a split feasibility problem. Inverse Probl. 2011, 27, 015007. [CrossRef]

16. Yang, Q. On variable-step relaxed projection algorithm for variational inequalities. J. Math. Anal. Appl. 2005, 302, 166-179. [CrossRef]

17. Zhao, J.; Zhang, Y.; Yang, Q. Modified projection methods for the split feasibility problem and multiple-sets feasibility problem. Appl. Math. Comput. 2012, 219, 1644-1653. [CrossRef]

18. Bnouhachem, A.; Noor, M.A. Three-step projection method for general variational inequalities. Int. J. Mod. Phys. B. 2012, 26, 1250066. [CrossRef]

19. Cordero, A., Hueso, J.L., Martinez, E., Torregrosa, J.R. Efficient three-step iterative methods with sixth order convergence for nonlinear equations. Numer. Algorithms 2010, 53, 485-495. [CrossRef]

20. Noor, M.A.; Noor, K. I. Three-step iterative methods for nonlinear equations. Appl. Math. Comput. 2006, 183, $322-327$.

21. Noor, M.A.; Yao, Y. Three-step iterations for variational inequalities and nonexpansive mappings. Appl. Math. Comput. 2007, 190, 1312-1321. [CrossRef]

22. Phuengrattana, W.; Suantai, S. On the rate of convergence of Mann, Ishikawa, Noor and SP-iterations for continuous functions on an arbitrary interval. J. Comput. Appl. Math. 2011, 235, 3006-3014. [CrossRef]

23. Rafiq, A.; Hussain, S.; Ahmad, F.; Awais, M.; Zafar, F. An efficient three-step iterative method with sixth-order convergence for solving nonlinear equations. Int. J. Comput. Math. 2007, 84, 369-375. [CrossRef]

24. Bauschke, H.H.; Combettes, P.L. Convex Analysis and Monotone Operator Theory in Hilbert Spaces; Springer: London, UK, 2011. 
25. Bauschke, H.H.; Combettes, P.L. A weak-to-strong convergence principle for Fejér-monotone methods in Hilbert spaces. Math. Oper. Res. 2001, 26, 248-264. [CrossRef]

26. He, S.; Yang, C. Solving the variational inequality problem defined on intersection of finite level sets. Abstr. Appl. Anal. 2013. [CrossRef]

(C) 2019 by the authors. Licensee MDPI, Basel, Switzerland. This article is an open access article distributed under the terms and conditions of the Creative Commons Attribution (CC BY) license (http:/ / creativecommons.org/licenses/by/4.0/). 